\title{
Entropy-Driven Phase Transition of Semiflexible Hard-Sphere Polymer Packings in Two and Three Dimensions $^{+}$
}

\author{
Daniel Martínez-Fernández, Clara Pedrosa, Miguel Herranz, Katerina Foteinopoulou, Nikos \\ Karayiannis and Manuel Laso \\ ETS Ingenieros Industriales/ISOM, Universidad Politécnica de Madrid, Madrid, Spain \\ + Presented at the Entropy 2021: The Scientific Tool of the 21st Century, 5-7 May 2021; Available online: \\ https://sciforum.net/conference/Entropy2021/.
}

Published: 5 May 2021

We study, at the atomic level, the behaviour of athermal, linear semiflexible polymers of tangent spheres in thin films of one-layer thickness (2-D systems) and bulk 3-D systems. We employ extensive Monte Carlo simulations [1] at progressively increased concentrations adopting the hard-sphere model to represent interactions between monomers. Extreme, plate-like confinement for thin films is realized through the presence of flat, parallel walls in one dimension with the inter-wall distance being equal to the diameter of the spherical monomers. Chain stiffness is controlled by a tuneable potential for the bending angles whose intensity dictates the rigidity of the polymer backbone. At very high values of bending intensity, the polymer model approaches that of freely-rotated chains and bending angles sample the whole range from acute to obtuse angles, reaching the limit of rod-like polymers. We study how packing density, chain length and stiffness affect the entropy-driven phase transition from initially disordered (random) to ordered (crystal) local and global structures in dense polymer packings in 2-D and 3-D systems and compare against fully flexible chains and monomeric counterparts [2]. To gauge local order, we employ the characteristic crystallographic element (CCE) norm, a descriptor, which can detect and quantify, with high precision, similarity to reference crystals in general atomic and particulate systems [3,4]. In all cases, we identify the critical volume fraction for the phase transition and gauge the established crystal morphologies.

\section{References}

1. Ramos, P.M.; Karayiannis, N.C.; Laso, M. Off-lattice simulation algorithms for athermal chain molecules under extreme confinement. J. Comput. Phys. 2018, 375, 918-934.

2. Karayiannis, N.C.; Foteinopoulou, K.; Laso, M. Spontaneous crystallization in athermal polymer packings. Int. J. Mol. Sci. 2013, 14, 332-358.

3. Karayiannis, N.C.; Foteinopoulou, K.; Laso, M. The characteristic crystallographic element norm: A descriptor of local structure in atomistic and particulate systems. J. Chem. Phys. 2009, 130, 074704.

4. Ramos, P.M.; Herranz, M.; Foteinopoulou, K.; Karayiannis, N.C.; Laso, M. Identification of Local Structure in 2-D and 3-D Atomic Systems through Crystallographic Analysis. Crystals 2020, 10, 1008.

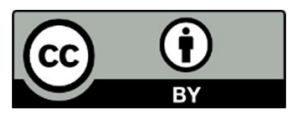

(C) 2021 by the authors. Licensee MDPI, Basel, Switzerland. This article is an open access article distributed under the terms and conditions of the Creative Commons Attribution (CC BY) license (http://creativecommons.org/licenses/by/4.0/). 